\title{
A Team-Based Approach to Providing Asthma Action Plans
}

\author{
Joyce E. Kaferle, MD, and Leslie A. Wimsatt, PhD
}

Background: National guidelines for treatment of asthma include recommendations for providing written asthma action plans (AAPs) to improve outcomes through patient self-management. However, AAP completion rates remain limited in practice.

Methods: We developed a team-based approach for the delivery of care to asthma patients in a primary care setting that involved integration of an electronic clinical quality management system and provision of written AAPs by registered nurses.

Results: The percentage of patients with AAPs increased after implementation of clinical reminders and nurse-led provision of written AAPs.

Conclusion: Proper training, use of an electronic clinical reminder system, and enhanced engagement of registered nurses can improve AAP completion rates in a team-based primary care setting. (J Am Board Fam Med 2012;25:247-249.)

Keywords: Asthma, Delivery of Health Care, Health Care Team, Patient Education

National guidelines for treatment of asthma include recommendations for providing written asthma action plans (AAPs) to support patient selfmanagement. ${ }^{1}$ However, research indicates that AAP completion rates often remain low despite such recommendations. ${ }^{2-4}$ Proactive, clinic-based approaches to asthma management have shown promise in increasing the use and ownership of AAPs, but they involve extensive provider training and/or health care worker time commitments. ${ }^{1,5}$

During the period 2005 to 2006, our academic health center reported low AAP completion rates (7.5\%; 884 of 11,785 patients) despite existing guidelines, AAP templates, and feedback from a quality improvement team. With no formal process in place for implementing clinic practice change, we piloted a team-based approach to increase the

This article was externally peer reviewed.

Submitted 29 April 2011; revised 1 September 2011; accepted 6 September 2011.

From the Department of Family Medicine, University of Michigan, Dexter, MI (JEK); and Department of Family Medicine, University of Michigan, Ann Arbor, MI (LAW).

Funding: none.

Conflict of interest: none declared.

Corresponding author: Joyce E. Kaferle, MD, Department of Family Medicine, University of Michigan, 7300 DexterAnn Arbor Road, Suite 1100, Dexter, MI 48130 (E-mail: jkaferle@med.umich.edu). number of patients with written AAPs at 5 community-based family medicine clinics. The clinics handled 1300 to 2500 patient visits per month with staffing levels of 8 to 17 physicians and 2 to 4 registered nurses (RNs). A clinical quality management system (CQMS, Crimson Care Registry, Ann Arbor, MI) provided reminders to the physician at the time of the office visit. The system was capable of monitoring management for patients with chronic conditions, producing lists of patients with persistent asthma, delivering evidence-based reminders for asthma management, and tracking provider-generated responses.

\section{Methods}

Our team-based approach involved CQMS reminder use and nurse engagement in AAP development to increase the number of asthma patients with written AAPs. Because merely educating physicians to provide AAPs does not necessarily lead to changes in practice, we used RNs and the CQMS. ${ }^{5}$ A family medicine physician, an outpatient pulmonary care $\mathrm{RN}$, and a family medicine outpatient $\mathrm{RN}$ (both RNs are certified as asthma educators) developed a 2-hour educational program for delivery to RNs, faculty, and residents. National guidelines for patient education shaped topic selection and AAP 
template design. Physicians received a 15-minute introduction to the new AAP prompts, $\mathrm{RN}$ referral process, AAP templates, and educational materials. ${ }^{4,5}$ Patients met with RNs for 1 hour or less after regularly-scheduled office visits as a follow-up to physician recommendations or in response to telephone invitations made by clinic staff using CQMS-generated patient lists.

After receiving an institutional review board nonregulated determination, we reviewed Blue Cross/ Blue Shield's Physician Group Incentive Program (PGIP) and CQMS data to examine AAP response rates between June 2007 and December 2009. We analyzed data descriptively and by simple linear regression over time (PASW Statistics 18.0; SPSS/ IBM, Chicago, IL).

\section{Results}

From 2006 to 2009, we educated 14 RNs, 30 residents, and 56 faculty. RNs wrote $\sim 98 \%$ of the AAPs based on physician and RN reports. Inclusion criteria for persistent asthmatics in the PGIP database somewhat differed over time because of changes in the reporting criteria of our internal health system, with a total of 10,071 to 11,686 recorded per year during the study period. Physician responses to the CQMS reminders remained high, ranging from 78\% (993 of $1276)$ in 2007 to $90 \%$ (1481 of 1647 ) in 2009 $(P=.121)$. Responses to AAP reminders included "completed today" (26\%), "ordered for upcoming visit" (9\%), "did not address due to more pressing health concern at visit" (13\%), "discussed/deferred" (49\%), and "refused" (3\%).
From June 2007 to December 2009, the total number of patients provided with written AAPs increased significantly over time at most family medicine clinics, and at a level that exceeded other primary care clinics within the health system (Figure 1). Clinic 3 served as the pilot site, and AAP rates remained highest there for the majority of the study period, only showing a slight decline in late 2009 after reaching saturation.

\section{Discussion}

In this study, AAP completion rates increased over time, with the largest and most rapid increases attributed to easily adopted clinical processes, as recommended by Ring et $\mathrm{al}^{5}$ following a systematic review of research about promoting AAP use. PGIP funding during this time was only used for development of the database and was not used for other asthma care improvement activities.

AAP completion rates rose throughout the institution, but the greatest improvements were shown in family medicine clinics that systematically implemented asthma training programs, use of clinical reminders, and enhanced RN engagement. Although our review was limited to retrospective analysis of data from a single family medicine department, the results suggest that proper RN and physician training coupled with the use of clinical reminder systems can significantly improve the number of AAPs provided to primary care patients.

Figure 1. Asthma action plan rates (cumulative \%) for patients with persistent asthma. Results are based on the Physician Group Incentive Program chart review. Physician Group Incentive Program data from December 2008 was not available. Average-0ther represents cumulative asthma action plan completion rates for the departments of internal medicine and pediatrics.

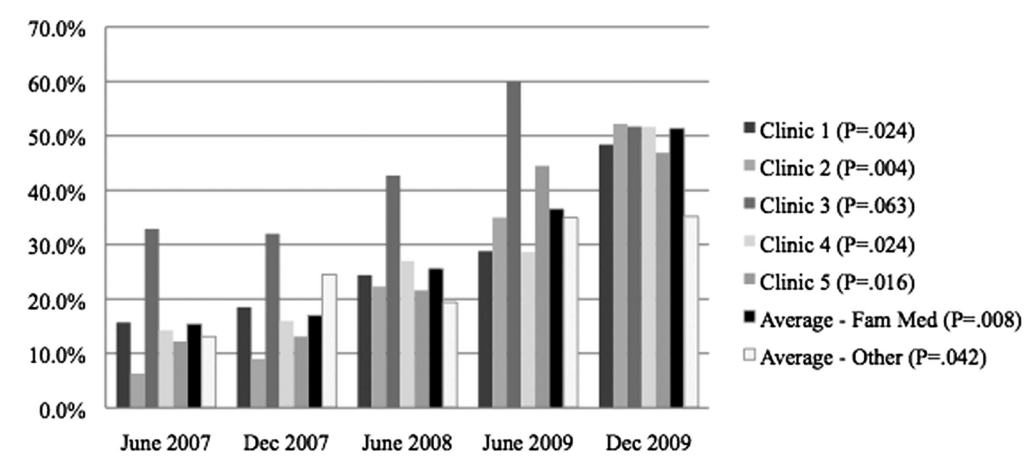




\section{Conclusion}

Practices with low AAP completion rates may find a team-based approach helpful in increasing the provision of written AAPs for patients with persistent asthma.

The authors gratefully acknowledge the support of Lee Green MD and Timothy Morris MS from the Department of Family Medicine at the University of Michigan for their assistance with data collection for this project.

\section{References}

1. National Heart, Lung and Blood Institute and National Asthma Education and Prevention Program. Guidelines for the diagnosis and management of asthma (EPR-3). Bethesda, MD: National Institutes of Health; 2007. Available at: http://www.nhlbi.nih. gov/guidelines/asthma/index.htm. Accessed March 30, 2011.

2. Grant EN, Moy JN, Turner-Roan K, Daugherty SR, Weiss KB. Asthma care practices, perceptions, and beliefs of Chicago-area primary-care physicians. Chest 1999;116:145S-54S.

3. Peters SP, Jones CA, Haselkorn T, Mink DR, Valacer DJ, Weiss ST. Real-world evaluation of asthma control and treatment (REACT): findings from a national web-based survey. J Allergy Clin Immunol 2007;119:1454-61.

4. Wisnivesky JP, Lorenzo J, Lyn-Cook R, et al. Barriers to adherence to asthma management guidelines among inner city primary care providers. Ann Allergy Asthma Immunol 2008;101:264-70.

5. Ring N, Malcom C, Wyke S, et al. Promoting the use of personal asthma action plans: a systematic review. Primary Care Resp J 2007;16:271-83. 